This item was submitted to Loughborough's Research Repository by the author.

Items in Figshare are protected by copyright, with all rights reserved, unless otherwise indicated.

\title{
Numerical analysis of composite structure with in-plane isotropic negative Poisson's ratio: effects of materials properties and geometry features of inclusions
}

\section{PLEASE CITE THE PUBLISHED VERSION}

http://dx.doi.org/10.1016/j.compositesb.2013.10.030

\section{PUBLISHER}

(C) Elsevier

\section{VERSION}

NA (Not Applicable or Unknown)

\section{PUBLISHER STATEMENT}

This work is made available according to the conditions of the Creative Commons Attribution-NonCommercialNoDerivatives 4.0 International (CC BY-NC-ND 4.0) licence. Full details of this licence are available at: https://creativecommons.org/licenses/by-nc-nd/4.0/

\section{LICENCE}

CC BY-NC-ND 4.0

\section{REPOSITORY RECORD}

Hou, Xiaonan, Hong Hu, and Vadim V. Silberschmidt. 2019. "Numerical Analysis of Composite Structure with In-plane Isotropic Negative Poisson's Ratio: Effects of Materials Properties and Geometry Features of Inclusions". figshare. https://hdl.handle.net/2134/25643. 


\title{
Numerical analysis of composite structure with in- plane isotropic negative Poisson's ratio: Effects of materials properties and geometry features of inclusions
}

\author{
Xiaonan Hou ${ }^{a}$, Hong $\mathrm{Hu}^{\mathrm{a}, *}$, Vadim Silberschmidt ${ }^{\mathrm{b}}$ \\ a Institute of Textiles and Clothing, Hong Kong Polytechnic University, Hong Kong \\ ${ }^{\mathrm{b}}$ Wolfson School of Mechanical and Manufacturing Engineering, Loughborough University, Loughborough, UK
}

A B S T R A C T

A novel composite structure with isotropic negative Poisson's ratio has been presented in our previous paper [1]. However, the previous study has only focused on the effects of random inclusions. In this work, an extended numerical study on the effects of materials properties and geometry features of inclusions is conducted in order to better understand the deformation mechanism and mechanical properties of this kind of composites. Using finite element method, the overall negative Poisson's ratio effects and mechanical properties of the composites are analyzed in terms of different factors including thickness and stiffness of inclusions with uniform and non-uniform struts. The structure optimization of the composites with better negative Poisson's ratio effect and mechanical performance are discussed based on the numerical calculated results. The study shows that the Poisson's ratio effects and mechanical properties of the composites are significantly affected by geometrical features, stiffness and boundary conditions of inclusions.

\author{
Keywords: \\ A. Smart materials \\ C. Finite element analysis (FEA) \\ B. Mechanical properties, Negative Poisson's \\ ratio
}

\section{Introduction}

The concept of material with a negative Poisson's ratio (NPR) has been studied for decades. Although many different structures have been suggested and analyzed, two limitations in the development of NPR materials still remain both in theoretical and practical aspects. The first limitation is that most of NPR structures are only studied in a two-dimensional space, and that their NPR effects are mostly limited to certain directions. This is true, for example, reentrant, chiral, star-shape structures and rotating units [2-6]. These structures, known as skeletal structures, are well studied, and most of them are periodic and often symmetric as well [7-15]. In these structures, the NPR effect only occurs along certain in-plane directions, and the concept of NPR is hardly used in 3D space. So far there are only few studies extending in-plane negative Poisson's ratio effect to 3D space [15-19] and only one structure with orthotropic NPR effect [15]. However, these 3D studies have only focused on certain cellular materials with highly heterogeneous porous microstructures. As stiffness of this type of materials is usually very low, their applications are limited.

Another limitation of present researches into NPR structures is linked to their manufacture and applications. Most of the

\footnotetext{
* Corresponding author. Tel.: +852 3400 3089; fax: +852 27731432

E-mail address: tchuhong@polyu.edu.hk (H. Hu).
}

developed theoretical models suggest materials with rather complex microstructures $[20,21]$ that are too difficult for manufacturing and employing in real engineering environment. So far, there are only a few successful NPR materials [22] which can be produced easily. However, the NPR effect of these materials still occurs in one direction due to their structural periodicity, and their deformation mechanism and mechanical properties have not been widely studied yet. Therefore, their applications are limited. These were the reasons for us to have developed a disordered structure having an in-plane isotropic mechanical NPR effect, a hardening mechanical behaviour and a relatively simple deformation mechanism in our previous study [1]. The composite structure previously suggested could not only be manufactured as an engineering material using existing techniques, but the proposed concepts can also easily be extended to 3D space. In this study, a 2D composite structure, which is formed by embedding re-entrant inclusions into a matrix with random locations and orientations, is developed. Different from the random orientation distribution function (ODF) for inclusions used in our previous models [1], the ODF of the models in this study is determined as a uniform distribution at the preprocessing stage. Thus, the developed models can be compared with each other. Based on the developed models, the deformation mechanism and mechanical performance of the composites are analyzed by modifying materials properties and geometrical parameters of inclusions. The results show that the NPR effect 
(a)

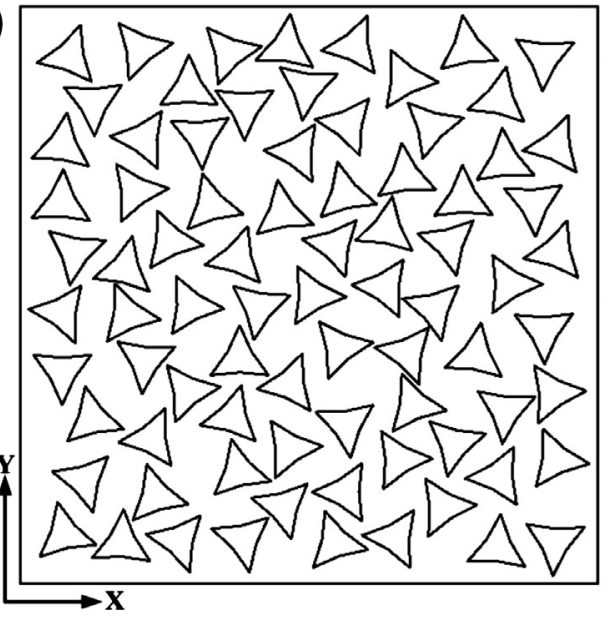

(b)

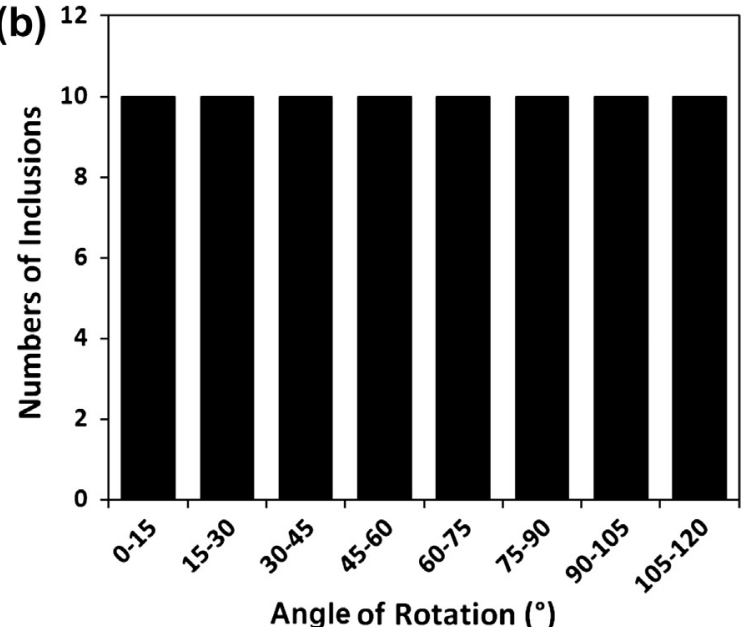

Fig. 1. Locations (a) and orientation distribution (b) of inclusions in FE models.

(a)

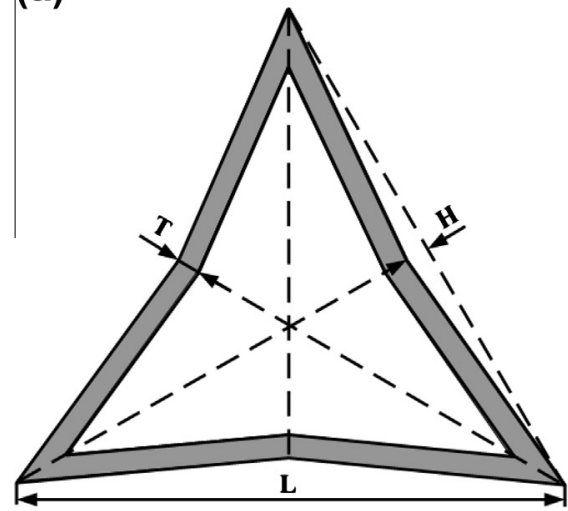

(b)

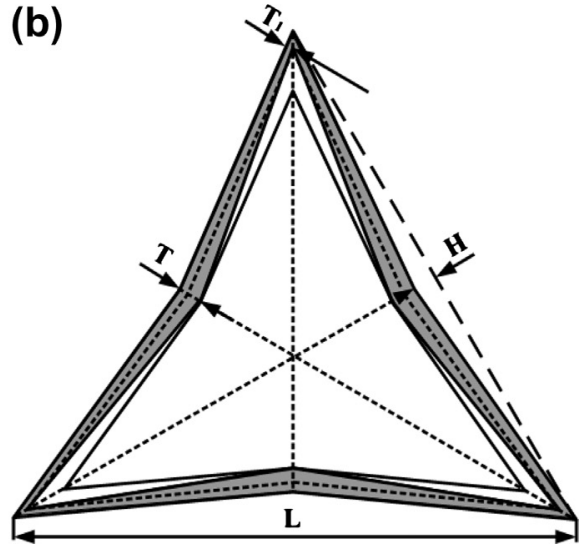

Fig. 2. Geometry models of re-entrant inclusions: (a) inclusion with uniform struts and (b) inclusion with non-uniform struts.

\section{Model-T/T-}

The thickness of wall of inclusion $\left(T / T_{1}\right)$.

$T: 0.1,0.2$ and 0.3

$T_{1}: 0.1,0.2$ and 0.3
Elastic modulus of wall of inclusion. $X: 100,60$ and 20

Fig. 3. Codes used for notation of finite element models.

and mechanical properties of the composites can be optimized by adjusting the material properties of constituents and geometry features of inclusions.

\section{Finite element model}

The main approach to develop FE models of NPR materials was discussed in the previous paper [1]. The FE models developed was to simulate a relatively large in-plane compression deformation (about 18\%) of the composite structures under static compression with consideration of geometry nonlinearity. The geometry models were developed using a programming language Python and the finite element analysis was performed by a commercial FE software
ABAQUS. In the composite structures, the re-entrant triangle inclusions were embedded in a square area $L \times L(L=100)$. The thickness of the square selected was 1 , and coordinates of the centre points of inclusions were randomly generated (Fig. 1a). To simplify the analysis, the orientation distribution of inclusions was determined as a uniform distribution in this study (Fig. 1b). Due to the geometrical features of the re-entrant triangle inclusions, the range of their rotation is $120^{\circ}$, and it was divided into 8 intervals. Within each interval, 10 random values of rotation angle were selected. In this way, the density of the inclusions in the models could be determined.

To analyze the effects of geometrical features of re-entrant inclusions on the overall deformation mechanism, inclusions with different struts were firstly developed. In Fig. 2, the grey area 
represents struts of inclusions whose re-entrant degree is controlled by parameter $H$, which was selected as 5 . Parameters $T$ and $T_{1}$ control the thickness of struts of the inclusions. For inclusions with uniform thickness, their struts thickness is adjusted by changing the value of $T$ (Fig. 2a). For inclusions with non-uniform thickness, $T$ and $T_{1}$ control the thickness of the central area and that of the pivoting area of the struts, respectively (Fig. 2b).

Another controlling factor of the composite structure is the materials properties of its constituents so that different material properties were defined to study their effects. To simplify the analysis, only linear elastic material properties were adopted at this stage of the research. It is no doubt that the material non-linearity of the constituents will affect the NPR behaviour of the composite structure and this factor will be included in our future study. In the present study, the elastic modulus and Poisson's ratio of the matrix for all models were defined by a given value $A$ and 0.3 , respectively. The elastic modulus of the struts was chosen among three different values: 100A, 60A and 20A. The Poisson's ratio of all the struts was selected as 0.3 as well. By changing the geometry variables and material properties, twelve different models were developed and analyzed. As shown in Fig. 3, special codes which could reflect different levels of the controlling parameters were adopted to note different models.

The boundary conditions of the models were defined in such a way that a compression test of composite materials could be simulated. The bottom of the units was fixed, with all the degrees of freedom constrained. A uniform displacement in the negative $y$ direction $(y=-20)$ was applied to the top of the modelled units to simulate static compression deformation. Meanwhile, the contacts between internal surfaces of the deformed inclusions were also taken into account in the simulation.

\section{Result and discussion}

\subsection{NPR effect}

The Poisson's ratio is defined as the negative ratio of the transverse strain to the axial strain. As the boundary conditions applied in the FE analysis was to simulate a real compression test in which two extremities of a composite structure are fixed with clippers, both the top and bottom surfaces of the composite structure could not shrink in the transverse direction under compression. Therefore, the transverse strain at the top and bottom surfaces should be zero. On the other hand, the micro-structure of the composite is not homogeneous. Because of these two reasons, the transverse displacements of nodes located at the left and right sides of the structure are not constant. In order to eliminate the effects of boundary conditions and non-homogeneity of the composite structure, only the averaged transverse strain calculated from the transverse displacements of nodes located at the left and right sides of the middle part of the structure was used to calculate the overall Poisson's ratio of the composite structure. The number of nodes selected at each side was 7 and the distance between two adjacent nodes was 2 . The averaged transverse strain was calculated by averaging the transverse strains obtained from these nodes. When the average strain was known, the overall Poisson's ratio $v$ of the composite structure could be obtained from the following relation.

$v=-\varepsilon_{\mathrm{t}} / \varepsilon_{\mathrm{a}}$

where $\varepsilon_{\mathrm{t}}$ and $\varepsilon_{\mathrm{t}}$ are the averaged transverse strain and overall compression strain of the composite structure, respectively.

Fig. 4 shows the evolutions of the Poisson's ratio with compression strain calculated from the models with the same materials properties but with different levels of uniform thickness of struts $(0.1,0.2$ and 0.3$)$. The change of the thickness of struts means
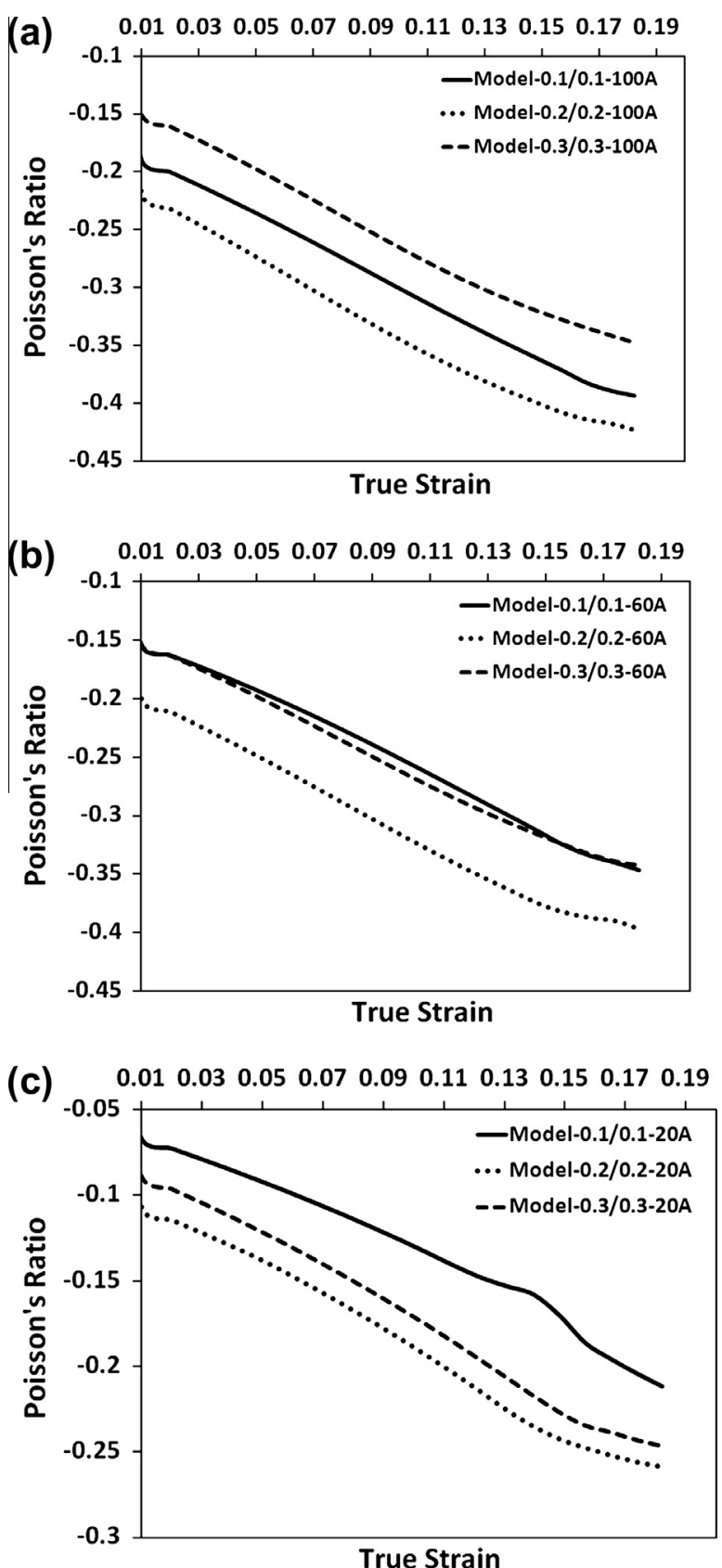

Fig. 4. Evolution of Poisson's ratio with strain for models with different stiffness of struts: (a) 100A; (b) 60A; and (c) 20A.

the change of their stiffness. Fig. 4a shows the results obtained when the elastic modulus of the struts was defined as $100 \mathrm{~A}$. It can be seen that the NPR effect increases with the increase of the compression strain. However, the effect of the thickness of struts on the NPR effect is not monotonous. The NPR effect increases when the thickness of struts increases from 0.1 to 0.2 , but considerably decreases when the thickness of struts becomes 0.3 . For instance, at the final strain of simulation (0.182), the NPR value of the model with 0.1 strut thickness is -0.394 . At the same strain level, the NPR value increases to -0.423 when the strut thickness changes to 0.2 . However, when the strut thickness increases to 0.3 , the NPR value reduces to -0.347 . Therefore, the model with 0.2 strut thickness has the highest NPR effect. Fig. $4 \mathrm{~b}$ shows the results obtained when the elastic modulus of the struts is reduced to 60A. A similar trend is also obtained. In this case, the model with 


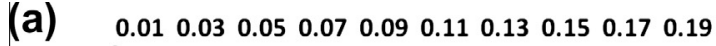
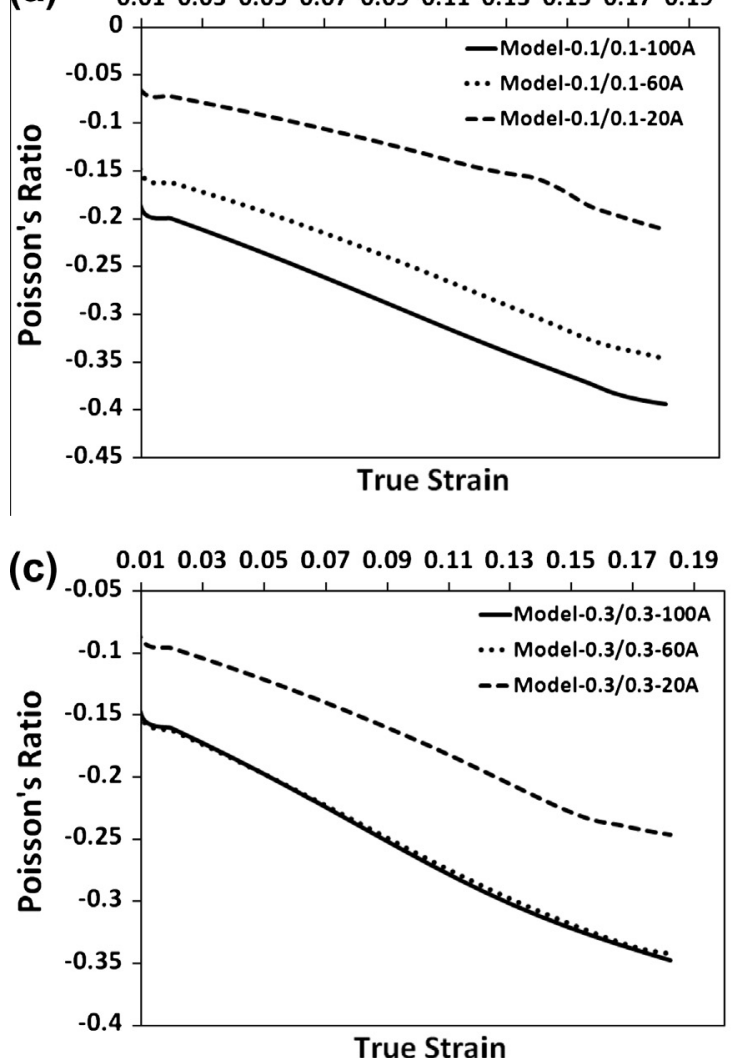

(b) $0.010 .03 \quad 0.050 .070 .090 .110 .13 \quad 0.150 .170 .19$
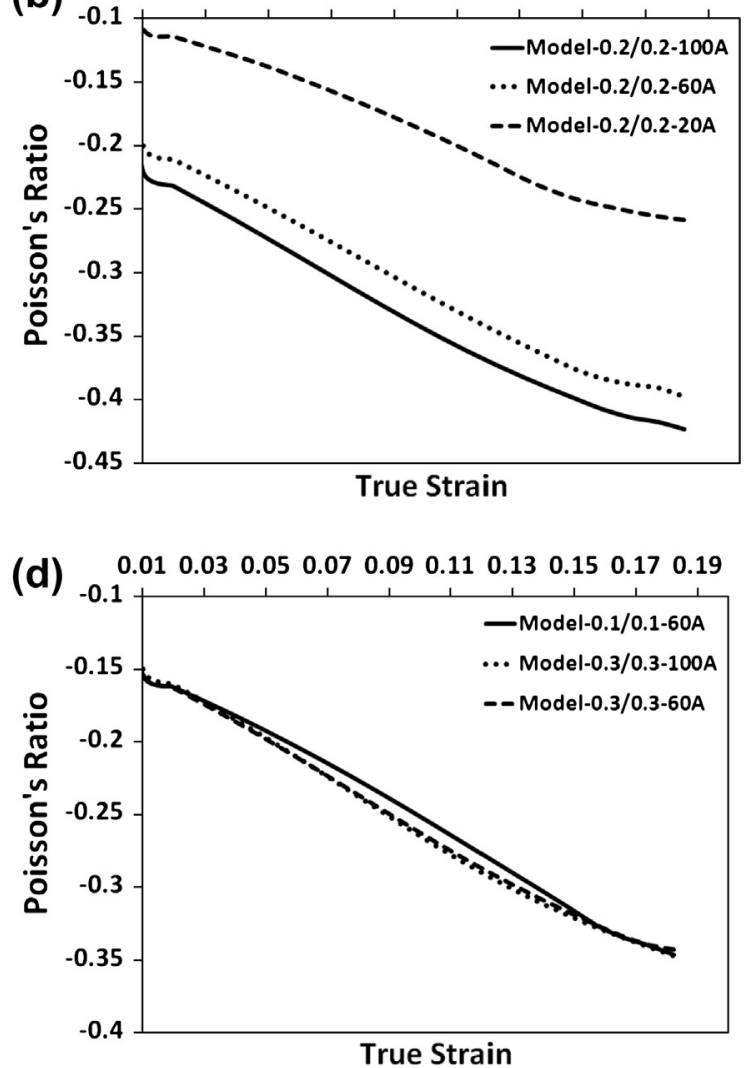

Fig. 5. Evolution of negative Poisson's ratio with strain for models with different thicknesses of struts: (a) 0.1 ; (b) 0.2 ; (c) 0.3 ; and (d) models with similar NPR.

0.2 strut thickness also has the highest NPR effect $(-0.397)$ at the final strain. However, the magnitude is $6.15 \%$ lower than that obtained with Model-0.2/0.2-100A due to reduction in stiffness of the struts. For the Model-0.1/0.1-60A and Model-0.3/0.3-60A, similar NPR levels are obtained. Their magnitudes at the final strain are -0.346 and -0.342 , respectively. However, the NPR value of the Model-0.1/0.1-60A is slightly lower than that obtained from the Model-0.3/0.3-60A when the strain is below 0.157. The Model$0.1 / 0.1-60 \mathrm{~A}$ has a higher NPR value when the strain exceeds this magnitude. Fig. $4 \mathrm{c}$ shows the results obtained when the elastic modulus of the struts is reduced to 20A. In this case, the NPR effect generally decreases due to low stiffness of struts. Again, the model with 0.2 strut thickness (Model-0.2/0.2-20A) has the highest NPR effect. Its NPR value at the final compression strain is -0.259 . However, different from the models with higher levels of stiffness of struts (100A and 60A), the model with 0.3 strut thickness (Model-0.3/0.3-20A) presents a higher NPR effect than the model with 0.1 strut thickness (Model-0.1/0.1-20A) does. These results show that the material properties of the inclusions significantly affect the NPR effect of the composites. But the effect is not independent of the geometrical features of inclusions. The opposite case is also true: the effect of geometrical features is not independent of material properties.

Fig. 5 shows the evolutions of the Poisson's ratio with compression strain calculated from the models with the same thickness of struts but with different material properties of inclusions. For the models with 0.1 and 0.2 strut thicknesses, the NPR effect increases with the increase of the stiffness of inclusions (Fig. 5a and b). However, when the thickness of struts becomes 0.3 , the trend changes (Fig. 5c). Interestingly, very close NPR effects are obtained for models with different material properties: Model-0.3/0.3-60A and Model-0.3/0.3-100A. This implicates that when the thickness of struts increases to a certain level, the overall structure loses its sensitivity to relatively low material properties. To summarize the above results, it has been found three models have the similar NPR effect (see Fig. 5d). According to the classic bending theory, the deflection of a beam is determined by its material properties, its cross-sectional moments of inertia, its length and boundary conditions. If a re-entrant inclusion is treated as a structure consisting of beams, it is easy to understand that the deformation mechanism of an inclusion is a collective phenomenon, highly dependent on its geometrical features, material properties and its boundary conditions. However, due to the non-uniformity of stress/strain distributions in the discontinuous composite structure, it is difficult to analyze a single inclusion as a representative cell. Because of this, the deformation mechanism also depends on locations and rotation angles of the inclusions that determine different boundary conditions for each inclusion.

\subsection{Mechanical properties}

Besides the NPR effect, the hardening effect of the composite structure is another important feature, which defines suitability of the composite to be used in real engineering applications. Figs. 6 and 7 present the calculated overall stress-strain relationships of the models with different material properties and geometrical features. Although the used material properties of constituents are linear elastic, the overall composite structure exhibits a non-linear response. For the models with same material properties and but with different thickness of struts (Fig. 6), the calculated stress increases with the increase of the thickness of struts of inclusions. The phenomenon generally agrees with the trend defined by the rule of mixtures of composites; the 
composite with a higher volume fraction of stiffer components has higher overall stiffness.

For the models with the same geometrical parameters but with different material properties (Fig. 7a-c), the levels of stress are determined by the material properties of the components: stiffer inclusions cause a stiffer overall mechanical behaviour. Fig. 7d presents the mechanical responses of the models with similar NPR as shown in Fig. 5d; different magnitudes of stresses are obtained. The results still comply with the trend that a stiffer mechanical behaviour is obtained for the model with a higher volume fraction of stiffer components with the same geometrical features. For the models with the same materials properties, the model with thicker struts of inclusions presents higher stiffness. Hence, this kind of composites with a certain degree of the NPR and a varied stiffness can potentially be produced for real-life applications, due to collective effects of the defined material properties and geometrical features.

\subsection{Optimized composite structures}

To optimize the NPR effect of the composite structures, three models (Model-0.2/0.1-100A, Model-0.3/0.1-100A and Model$0.3 / 0.2-100 \mathrm{~A}$ ) with non-uniform longitudinal thickness of struts were developed. The dimensions and shape of the struts are controlled by parameters $T$ and $T_{1}$ as shown in Fig. 2b. Since the highest NPR effect of the models with uniform struts was obtained from Model-0.2/0.2-100A (Fig. 4), it is used to compare with the models having non-uniform struts (Fig. 8a). The elastic modulus of struts in the models was defined as $100 \mathrm{~A}$, the same of the Model-0.2/0.2-100A. The results demonstrate that non-uniform struts could enhance the overall NPR effect of the composite structure. The Model-0.3/0.1-100A has the highest NPR effect among all the models. Its Poisson's ratio value is -0.447 at the final strain $(0.182)$, which is $5.67 \%$ higher than that obtained with the Model-0.2/0.2-100A. This means that thinner struts in the pivoting areas can enhance the rotational degree of freedom of the area and can improve the closing effect of the re-entrant inclusions. In Fig. 9, one inclusion at the same location is selected from the two models. The inclusion in the Model-0.3/0.1-100A tends to close earlier than that in the Model-0.2/0.2-100A, leading to a stronger NPR effect for the overall composite structure. Fig. 8b shows the stress-strain relationships of the models with high NPR effects; apparently, stress does not simply increase with the increase in the volume fraction of stiffer components. As shown in Table 1 , the volume fraction of struts in the Model$0.2 / 0.2-100 \mathrm{~A}$ is $4.60 \%$, which is slightly lower than that in the Model-0.3/0.1-100A (4.65\%). But the maximum stress in the former model is $8.61 \%$ higher than that obtained with the latter, which can be explained by differences in deformation mechanisms. The re-orientation mechanism of the struts is different, affecting the extent of the overall mechanical response.

Fig. 10 shows the relationship between the composite's modulus $(E)$ and strain calculated with the suggested finite element models. Here, the modulus $E$ is defined as the ratio of the value of stress to the value of strain of the overall composite structure. The modulus increases with the increase of compression strain, demonstrating a hardening effect of the composite structure. In general, the higher modulus is obtained for models with higher volume fractions of stiffer materials (Table 1 ). However, there is one exception in our study. Model-0.2/0.2-100A and Model-0.3/ $0.1-100 \mathrm{~A}$ have close volume fractions of struts, which are $4.60 \%$ and $4.65 \%$, respectively, but the modulus of Model-0.2/0.2-100A is apparently higher than that of Model-0.3/0.1-100A. The phenomenon means that the rule of mixtures cannot adequately explain the mechanical performance of the developed discontinuous composite structure with rather complex internal deformation
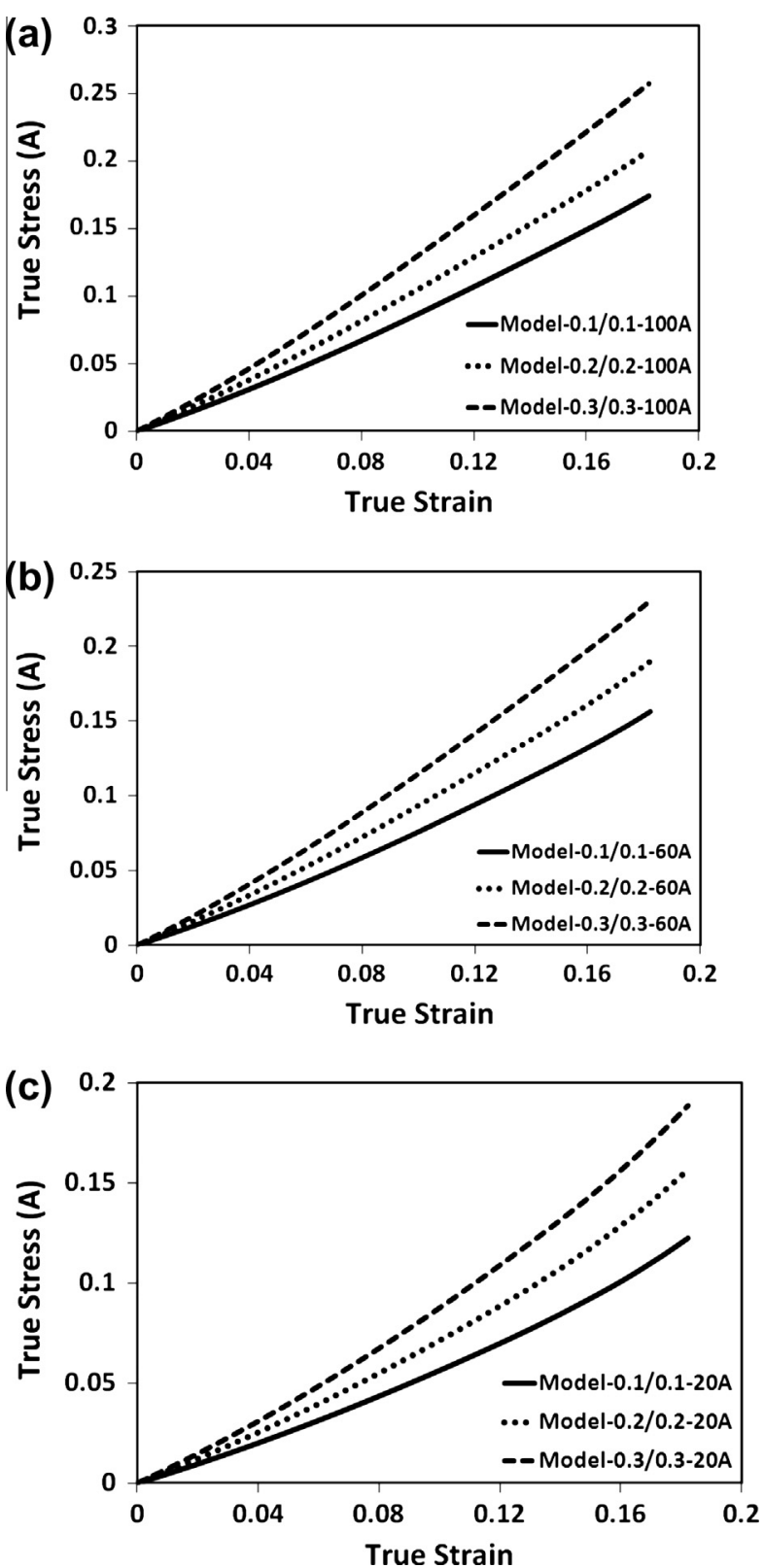

Fig. 6. Stress-strain relationships for models with different material properties of struts: (a) 100A; (b) 60A; and (c) 20A

mechanisms. The presence of compressible voids in the structure results in violation of the rule of mixtures.

In the developed composite structure, the overall volume consists of volume of inclusions $\left(V_{\mathrm{i}}\right)$, volume of voids $\left(V_{\mathrm{v}}\right)$ and volume of matrix $\left(V_{\mathrm{m}}\right)$. To estimate its hardening effect, an effective modulus was determined based on the theory of the rule of mixtures by considering the effect of voids. It is an equivalent modulus of a composite material with internal voids.

First, it is assumed that there is a continuous composite material only consisting of strut and matrix materials. Its height is $L=100$, thickness is 1 and volume is $V^{\prime}$, which is given by the following equation:

$V^{\prime}=V_{\mathrm{i}}+V_{\mathrm{m}}$

Since there are no voids in this continuous composite structure, the low-bound modulus of the composite $E^{\prime}$ could be easily calculated from Eq. (3) of the rule of mixtures. 

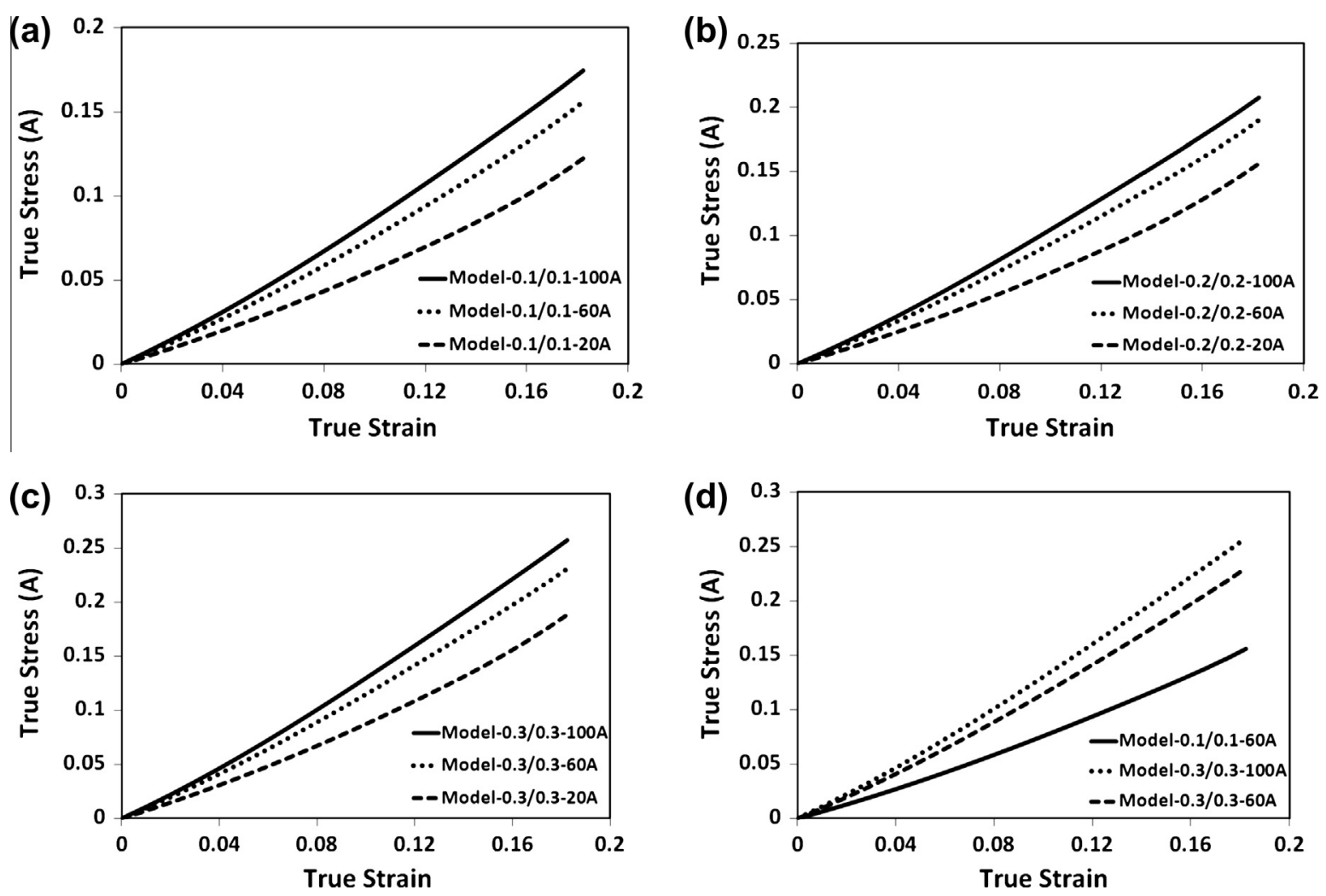

Fig. 7. Stress-strain relationships for models with different thickness of struts: (a) 0.1 ; (b) 0.2 ; (c) 0.3 ; and (d) models with similar NPR.
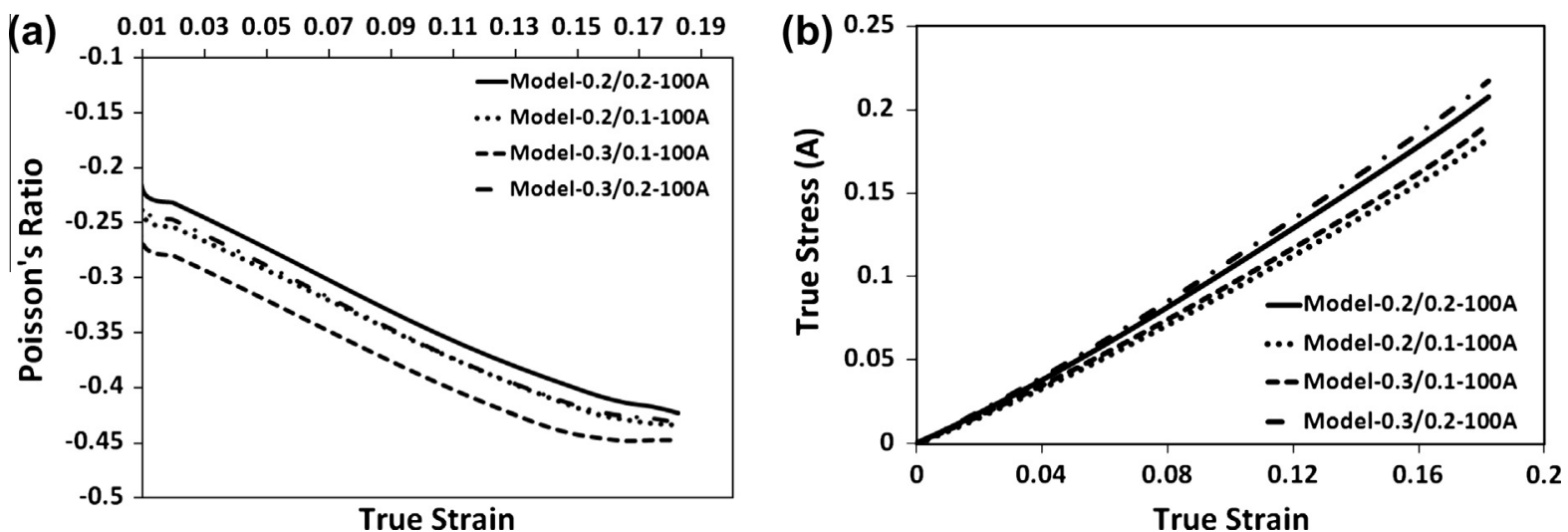

Fig. 8. Mechanical performance of optimized structures: (a) evolution of Poisson's ratio with strain and (b) stress-strain relationship.

$E^{\prime}=\left(E_{\mathrm{i}} * E_{\mathrm{m}}\right) /\left(E_{\mathrm{i}} *\left(V_{\mathrm{m}} / V^{\prime}\right)+E_{\mathrm{m}} *\left(V_{\mathrm{i}} / V^{\prime}\right)\right)$

where $E_{\mathrm{i}}$ is the elastic modulus of the material used for struts of inclusions, $E_{\mathrm{m}}$ is the modulus of the matrix. So, for a given strain $\varepsilon$, the theoretical reaction force $F$ of this continuous composite could be calculated from the following equation:

$F=E^{\prime} * \varepsilon * S^{\prime}$

where $S^{\prime}$ is the cross-sectional area of the continuous composite.

Then, the same volume of the materials is used to develop a discontinuous composite structure with added voids $\left(V_{\mathrm{v}}\right)$. The discontinuous composite has the same voids volume of the developed NPR composite structure and the same height of the continuous composite. When the reaction force $F$ and strain $\varepsilon$ of both the discontinuous and continuous composites are supposed to be the same, the effective modulus can be obtained from the following equation:

$E_{\mathrm{e}}=E^{\prime} *\left(S^{\prime} / S\right)=E^{\prime} *\left(V^{\prime} /\left(V^{\prime}+V_{\mathrm{v}}\right)\right)$

where $E_{\mathrm{e}}$ and $S$ are the effective modulus and cross-sectional area of the discontinuous composite structure, respectively.

Table 2 compares the effective modulus $E_{\mathrm{e}}$ and the modulus $E$ calculated from the developed FE models. The magnitudes of $E$ are apparently higher than those of $E_{\mathrm{e}}$ for all the models. The highest effective modulus is obtained for Model-0.3/0.3-100A, since the structure contains the highest volume fraction of the stiffer constituent. Comparing the moduli calculated with FE models (Fig. 10), it can be found that the models with much lower volume fractions of stiffer material even have moduli higher than $E_{\mathrm{e}}$. And it is easily deduced that the stiffness of the developed discontinuous 
Deformed

Model-0.3/0.1-100A

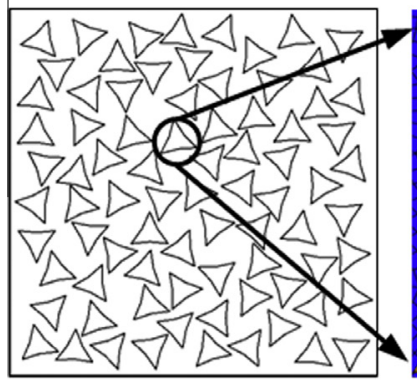

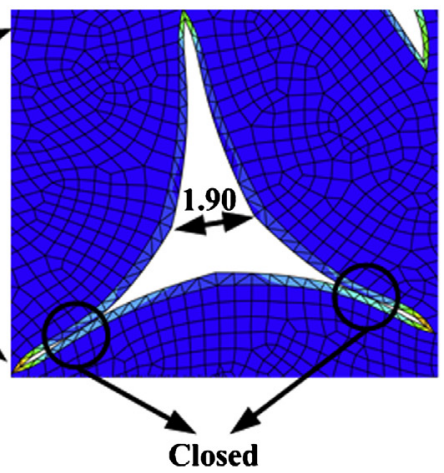

Deformed

Model-0.2/0.2-100A

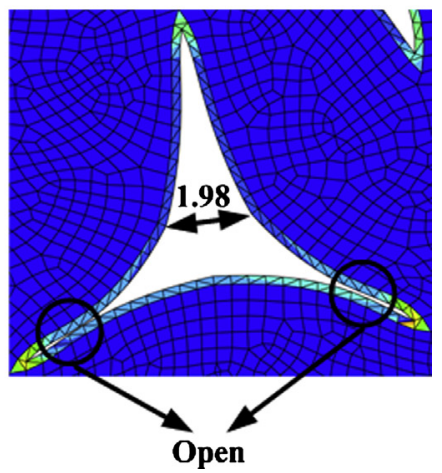

Fig. 9. Local deformation of inclusion with different struts (global strain $=0.182$ ).

Table 1

Volume fraction of inclusions of different models.

\begin{tabular}{|c|c|c|c|c|c|c|}
\hline Model code & Model $0.1 / 0.1$ & Model 0.2/0.2 & Model 0.3/0.3 & Model $0.2 / 0.1$ & Model $0.3 / 0.1$ & Model $0.3 / 0.2$ \\
\hline Volume fraction of inclusion material (\%) & 2.35 & 4.60 & 6.75 & 3.50 & 4.65 & 5.70 \\
\hline
\end{tabular}

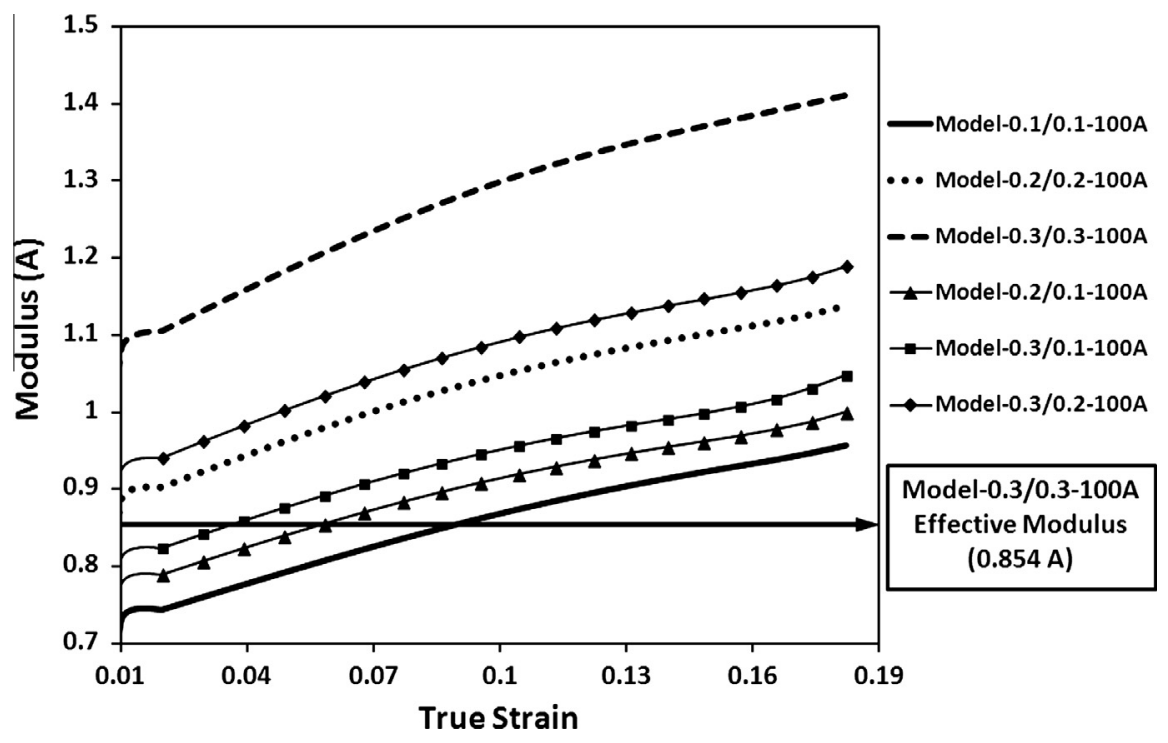

Fig. 10. Evolution of modulus with strain for composite structures with high negative Poisson's ratios.

Table 2

Mechanical properties of models with relatively high negative Poisson's ratio effect.

\begin{tabular}{|c|c|c|c|c|c|c|}
\hline Model code & $\begin{array}{l}\text { Model-0.1/0.1- } \\
100 \mathrm{~A}\end{array}$ & $\begin{array}{l}\text { Model-0.2/0.2- } \\
100 \mathrm{~A}\end{array}$ & $\begin{array}{l}\text { Model-0.3/0.3- } \\
100 \mathrm{~A}\end{array}$ & $\begin{array}{l}\text { Model-0.2/0.1- } \\
100 \mathrm{~A}\end{array}$ & $\begin{array}{l}\text { Model-0.3/0.1- } \\
100 \mathrm{~A}\end{array}$ & $\begin{array}{l}\text { Model-0.3/0.2- } \\
100 \mathrm{~A}\end{array}$ \\
\hline Effective modulus $\left(E_{\mathrm{e}}\right)$ & $0.761 \mathrm{~A}$ & $0.808 \mathrm{~A}$ & $0.854 \mathrm{~A}$ & $0.785 \mathrm{~A}$ & $0.809 \mathrm{~A}$ & $0.820 \mathrm{~A}$ \\
\hline $\begin{array}{l}\text { Modulus calculated from FE models at strain of } \\
0.182(E)\end{array}$ & $0.957 \mathrm{~A}$ & $1.139 \mathrm{~A}$ & $1.411 \mathrm{~A}$ & $1.000 \mathrm{~A}$ & $1.048 \mathrm{~A}$ & $1.190 \mathrm{~A}$ \\
\hline
\end{tabular}

composite structures could be much higher when they are further compressed. With closure of inclusions, the densification effect will significantly increase the overall stiffness. These results show that the developed discontinuous composite structure has reasonable mechanical properties as a composite with internal voids. And the use of component materials is efficient.

\section{Conclusions and remarks}

To analyze the suggested composite structure with an in-plane negative Poisson's ratio, finite element models with different material properties and geometrical features of inclusions are developed. Two important characteristics of such composite 
structures, i.e., the NPR effect and the hardening effect, are studied based on the FE models. According to the computational results, the factors affecting mechanical performances of the composite structure are summarized and discussed.

1. The material properties of constituents of the composite structure significantly affect the NPR effect of the overall composite material. However, the effect is very complicated and dependent on the effect of dimensions and geometrical features of inclusions. For example, the structure with inclusions formed with non-uniform struts can have a stronger NPR effect than that with uniform struts. To obtain an optimized composite structure with a stronger NPR effect, both the material properties and geometrical parameters have to be considered together.

2. The developed structures have rather interesting overall mechanical properties, especially the hardening effect. Generally, for the composites with the same microstructures, higher material properties implicate higher overall stiffness. The same is true for higher volume fractions of stiffer components. But in the studied structures, the observed trend is not in accord with the classic rule of mixtures, since voids exist in the composites and struts undergo re-orientations at local scales during overall compression.

3. Although the deformation mechanism and mechanical properties of the suggested composite structures with NPR are nontrivial, their mechanical performance can still be optimized by adjusting the important factors. By using finite element method, the optimized structure can be developed.

Based on the FE analysis in this study, a better understanding of the developed composite structure is obtained. For a composite structure with determined locations and orientation distributions of inclusions, the main factors affecting their mechanical performance are material properties of the components, shape, dimensions and detailed geometrical features of the inclusions. Besides, it is shown that the NPR and mechanical properties of this kind of composite structures could be further optimized by changing locations and orientation distributions of inclusions, which are worth of a further study.

\section{Acknowledgements}

The authors would like to thank the funding support from the Research Grants Council of Hong Kong Special Administrative Region Government in the form of a GRF project (Grant Number 516510).

\section{Reference}

[1] Hou X, Hu H, Silberschmidt V. A novel concept to develop composite structures with isotropic negative Poisson's ratio: effects of random inclusions. Compos Sci Technol 2012;72(15):1848-54.

2] Prawoto Y. Seeing auxetic materials from the mechanics point of view: a structural review on the negative Poisson's ratio. Comput Mater Sci 2012;58:140-53.

[3] Yang W, Li Z, Shi W, Xie B, Yang M. Review on auxetic materials. J Mater Sci 2004;39:3269-79.

[4] Kaminakis NT, Stavroulakis GE. Topology optimization for compliant mechanisms, using evolutionary-hybrid algorithms and application to the design of auxetic materials. Composites: Part B 2012:43:2655-68.

[5] Scarpa F, Blain S, Lew T, Perrott D, Ruzzene M, Yates JR. Elastic buckling of hexagonal chiral cell honeycombs. Composites: Part A 2007;38:280-9.

[6] Pozniak AA, Smardzewski J, Wojciechowski KW. Computer simulations of auxetic foams in two dimensions. Smart Mater Struct 2013;22:1-11.

[7] Yang DU, Lee S, Huang FY. Geometric effects on micropolar elastic honeycomb structure with negative Poisson's ratio using the finite element method. Finite Elem Anal Des 2003;39:187-205.

[8] Alderson A, Alderson KL. Auxetic materials. J Aerospace Eng 2007;221(4):565-75.

[9] Mitschke H, Schwerdtfeger J, Schury F, Stingl M, Korner Cl, Singer RF, et al. Finding auxetic frameworks in periodic tessllations. Adv Mater 2011;23:2669-74.

[10] Fozdar DY, Soman PP, Lee JW, Han LHl, Chen S. Three-dimensional polymer constructs exhibiting a tunable negative Poisson's ratio. Adv Funct Mater 2011;21:2712-20.

[11] Xu B, Arias F, Brittain ST, Zhao X, Grzybowski B, Torquato S, et al. Making negative Poisson's ratio microstructures by soft lithography. Adv Mater 1999;11(14):1186-9.

[12] Zhang Z, Hu H, Liu S, Xu B. Study of an auxetic structure made of tubes and corrugated sheets. Phys Status Solidi (b) 2013:1-6. http://dx.doi.org/10.1002/ pssb. 201248349.

[13] Ge Z, Hu H. Innovative three-dimensional fabric structure with negative Poisson's ratio for composite reinforcement. Textile Res J 2013;83(5):543-50.

[14] Fraldi M, Carannante F, Nunziante L. Analytical solutions for n-phase functionally graded material cylinders under de saint venant load conditions: homogenization and effects of Poisson ratios on the overall stiffness. Compos B Eng 2013;45(1):1310-24.

[15] Babaee S, Shim J, Weaver JC, Chen ER, Patel N, Bertoldi K. 3D soft metamaterials with negative Poisson's ratio. Adv Mater 2013. http:// dx.doi.org/10.1002/adma.201301986.

[16] Lakes R. Negative Poisson's ratio materials. Science 1987;238:551.

[17] Lakes R. Foam structures with a negative Poisson's ratio. Science 1987;235(4792):1038-40.

[18] Lakes RS, Witt R. Making and characterizing negative Poisson's ratio materials. Int J Mech Eng Educ 2002;30(1):50-8.

[19] Choi JB, Lakes RS. Nonlinear analysis of the Poisson's ratio of negative Poisson's ratio foams. J Compos Mater 1995;29(1):113-28.

[20] Shufrin I, Pasternak E, Kyskin AV. Planar isotropic structures with negative Poisson's ratio. Int J Solids Struct 2012:49:2239-53.

[21] Horrigan EJ, Smith CW, Scarpa FL, Gaspar N, Javadi AA, Berger MA, et al. Simulated optimisation of disordered structures with negative Poisson's ratios. Mech Mater 2009;41(8):919-27.

[22] Ma Z, Bian H, Sun C, Hulbert GM, Bishnoi K, Rostam-Abadi F. Functionallygraded NPR material for a blast-protective deflector. In: Proceedings of the 2009 ground vehicle systems engineering and technology symposium (GVSETS); 2010. p. 1 